\title{
Vehicle Accident Recognition \& Reporting System using Accelerometer in Android
}

\author{
V.K. Pooja, Amit.V. Kachavimath, Shashidhar Kuri and R. Vanishree Kinnnal
}

\begin{abstract}
The usage of vehicles is rapidly increasing and at the same time the occurrence of accident is also increased. Hence, the value of human life is ignored. No one can prevent the accident, but can save their life by expediting the ambulance to the hospital in time. The objective of this scheme is to minimize the delay caused by traffic congestion and to provide the accident information for smooth flow of emergency vehicles. These lives could have been saved if medical facilities are provided at the right time. This paper implies system which is a solution to this drawback. Accelerometer sensor can be used in car security system to sense vibrations in vehicle and GPS to give location of vehicle, so dangerous driving can be detected. When accident occurs, Accelerometer will detect signal and will send message to server. The message contains information of accident location, images and vehicle details. The message will be sent to preprogrammed numbers such as ambulance, friends, relatives etc via GSM.
\end{abstract}

Keywords--- Android, GPS (Global Positioning System), GSM(Global Service for Mobile Applications), Accelerometer.

\section{INTRODUCTION}

A Considerable work has been done in this area for the implementation of Accident alert system using android phones as alternatives to the detection of accident using the hardware enabled devices to improve both usability and efficiency issues which are explained in details below. It involves the detail study of existing system and limitations. The most common approach of accident detection was by human observation or by human communication. This approach is considered to be the traditional approach of accident detection. At these criteria, we cannot detect where the accident has occurred and hence no information related to it, leading to the death of an individual if there isn't any human intervention. To address this problem, some researchers have developed accident detection system that uses hardware configured with specific sensors for accident detection.

The high demand of automobiles has also increased the traffic hazards and the road accidents. There is high risk for people's life. This is because of the lack of accident detection

V.K. Pooja, Student, Computer Science, SKSVMACET, Laxmeshwar, India. E-mail:pooja.kachavimath@gmail.com

Amit.V. Kachavimath, Assistant Professor, Department of MCA, KLE Technological University, Hubli, India. E-mail:amit.kachavimath@gmail.com

Shashidhar Kuri, Student, Department of MCA, BVBCET, Hubli, India. Skshashidhar9@yahoo.in

R. Vanishree Kinnal, Department of MCA, BVBCET,Hubli, India. E-mail: vrkinnal@gmail.com

DOI:10.9756/BIJSESC.8255 at the right time and notification to the authorized emergency contacts, which will help in saving the valuable lives. An automatic alarm device for vehicle accidents is introduced. This design is a system which can detect accidents in significantly less time and sends the basic information to the monitoring server within a few minutes covering geographical coordinates, the time and sensor details in which a vehicle accident had occurred. Our application will use a common android phone which will immediately notify the concerned people about the accident.

As shown in the above Fig 1 when the accident occurs, the system will detect that. After that the system will capture the real-time image using the camera, GPS location and level of impact from the scenario. This collected information will be combined in an SMS and email then it will be sent to default set of emergency contacts.

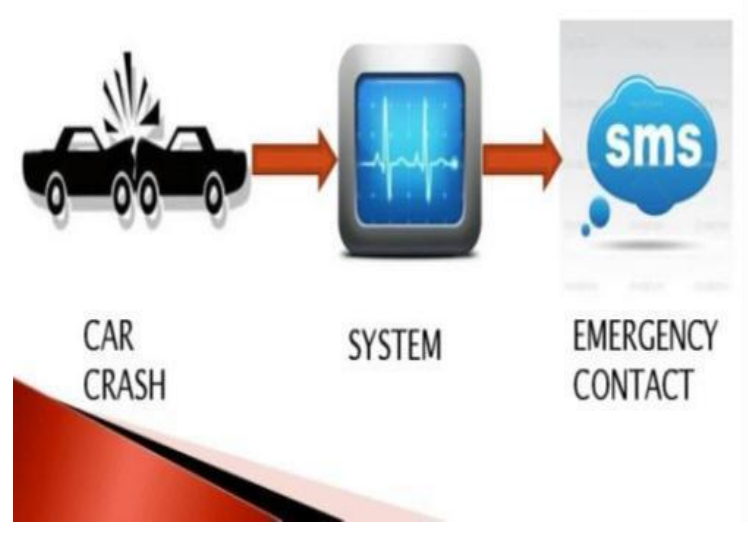

Figure 1: Overview of Application

\section{PROPOSED SYSTEM}

Accident Alert System using android would be more convenient, relatively secure and utilize fewer resources .In case of the proposed system all the facilities provided are same as in the case of existing system but the proposed system provides all the functionalities in a single device with less cost. This system is developed and implemented to track the vehicle and detect the accident very quickly and easily so that to proceed with the further action items. Proposed system is implemented in order to deliver the most effective and efficient system to users or the concerned once. It's less time consuming as it is online. Accident Alert System using android can provide better service to the users at less cost to the Commonwealth and can make more attractive place to live. The system is flexible enough to be modified easily and overcomes the drawback of existing system. 
The Android platform provides several sensors that let you monitor the motion of a device. Two of these sensors accelerometer and gyroscope are always hardware-based and these sensors gravity, linear acceleration and rotation vector sensors can be either hardware-based or software-based. Some devices have the software-based sensors derive their data from the accelerometer and magnetometer, but on other devices they may also use the gyroscope to derive their data. Most Android-powered devices have an accelerometer, and many now include a gyroscope. The availability of the softwarebased sensors is more variable because they often rely on one or more hardware sensors to derive their data.

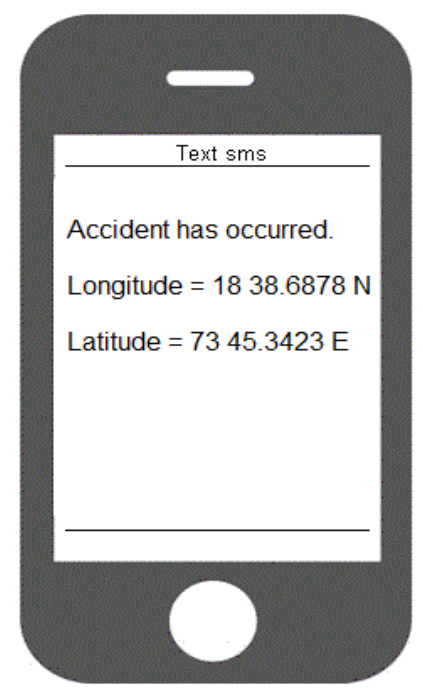

Figure 2: Snapshot of Accident Alert System Using Android

Based on the drawbacks of the existing system, we need to design the optimal solution for accident detection using single hardware and software configured device. This helps in identifying and tracking of accident in most effective and less time consuming approach. In order to achieve this we have come up with the idea of Accident Alert System using Android, which reduces human interventions considerably. This is more efficient and ensures maximum utilization of the system for detection and tracking of the vehicle without any manual or multiple external hardware involvement. The idea is represented in a block diagram shown in Fig 2.

The authorized user has to login, and get authenticated from the centralized server. The mobile device which uses the application must be ensured that it has General Packet Radio Service (GPRS) being enabled. Once the login is successful, try to get the current location of the user to confirm that GPS is enabled. Authenticated android enabled mobile device will be transmitting GPS details, such as latitude and longitude information to the monitoring unit for every regular time intervals.

GPS is used in vehicles for both tracking and navigation. Tracking systems enable a base station to keep track of the vehicles without the intervention of the driver where, as navigation system helps the driver to reach the destination. Whether navigation system or tracking system, the architecture is more or less similar. When an accident occurred in any place then GPS system tracks the position of the vehicle and sends the information to the particular person through GSM by alerting the person through SMS or by a call.

System proactively monitors the occurrence of accident using the in-build accelerometer sensor. When the accelerometer sensor values exceeding the threshold value, mobile device will notify the prediction of accident and transmits the data to the monitoring server and will process the data and identify the user details. It will be able to forward the auto generated text message to the default set of emergency contacts related to the user and also to the rescue team.

\section{SySTEM ARCHITECTURE}

The architectural design process is concerned with establishing a basic structural framework for a system. The Large systems are always decomposed into sub-systems that provide some related set of services. The initial design process of identifying these sub-systems and establishing a framework for sub-system control and communication is called Architecture design and the output of this design process is a description of the software architecture. The Fig 3 depicts proposed System Architecture and how the different components are connected \& interacted with each other.

Modern mobile phones come with a variety of sensors that automate or easy many of our daily tasks. This field takes into account the presence of an accelerometer, a gyroscope, compass and a barometer.

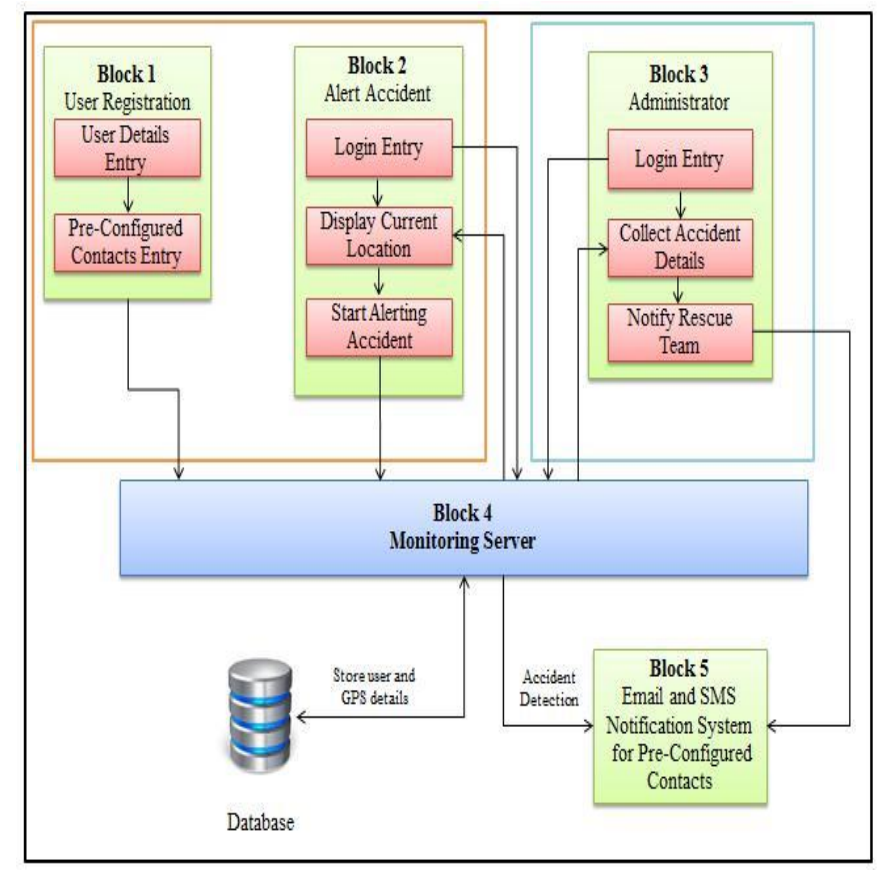

Figure 3: Proposed System Architecture

Accelerometers in mobile phones are used to detect the orientation of the phone. The gyroscope or gyro for short, adds an additional dimension to the information supplied by the accelerometer by tracking rotation or twist. An accelerometer measures linear acceleration of movement, while a gyro on the other hand measures the angular rotational velocity. Both sensors measure rate of change. In practice it means that an accelerometer will measure the directional movement of a 
device but will not be able to resolve its lateral orientation or tilt during that movement accurately unless a gyro is there to fill in that info. With an accelerometer you can either get a really "noisy" info output that is responsive or you can get a "clean" output that's sluggish. But after combining the 3-axis accelerometer as shown in the figure 4 with a 3 -axis gyro we get an output that is both clean and responsive in the same time."

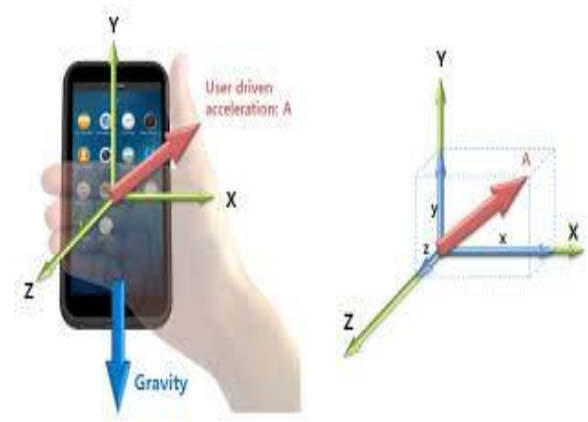

Figure 4: Working of Accelerometer Sensor

\section{SimUlation RESULTS}

The system can be designed to represent the tracking of the location for a particular vehicle. The get my location can be used to fetch the current latitude and longitude .The start tracking will be used to track the location of vehicle as it moves from source to destination. The values of latitude and longitude of the vehicle will be upgraded in the server for every specific regular intervals of time.

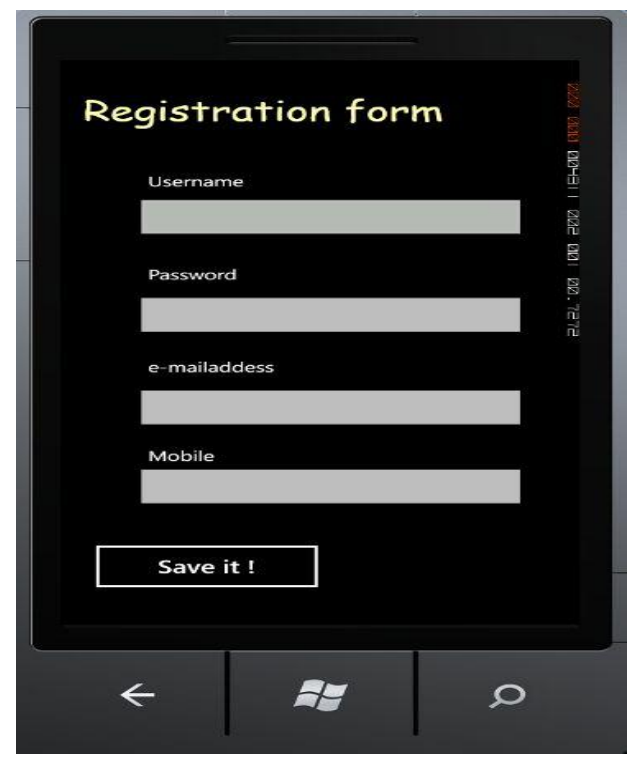

Figure 5: Registration Form

During the registration process the user will enter the emergency contact details with their email id. In case of accident occurred then along with the images of the vehicle, latitude and longitude details will be sent to emergency contact persons. The contact persons after referring can save the life of their esteemed ones.

\section{CONCLUSION}

With the advent of science and technology in every walk of life the importance of vehicle safety has increased and the main priority is being given to reduce the alarming time when an accident occur, so that the wounded lives can be attended in lesser time by the rescue team. This system provides the solution which has the advantages of low cost, portability, small size and easy extendable. This system can overcome the problems of lack of automated system for accident location detection. Consequently the time for searching the location is reduced and the person can be treated as soon as possible which will save many lives. The accident can be detected by both Accelerometer sensor and location can be detected using GPS which will give the accurate information. The system will process the data, as soon as input is received from the sensor and the alarm is notified and message is sent through the GSM module. The accident location automatic detection will help us to provide security to the vehicles and to the lives of the people. The high priority is given to the lives of the people. Hence, this project provides a feasible solution to traffic hazards and it gives security to vehicle and reduces loss of valuable lives and property.

\section{REFERENCES}

[1] Wang Wei, "Embedded Microcontroller MC9S08AW60 Principles and Application", Beijing Aerospace University Press, 2008.

[2] Y. Zhao, "Mobile phone location determination and its impact on Intelligent transportation systems".

[3] V. Dhana Raj, G. Vasu and S. Kanaka Durga, "ARM-7 Based Semi Autonomous Vehicle", IJRCCT, Vol. 1, No. 4, Pp. 160-163, 2012.

[4] Y.W. Bai, S.C. Wu and C.L. Tsai, "Design and implementation of a fall monitor system by using a 3-axis accelerometer in a smart phone", IEEE Transactions on Consumer Electronics, Vol. 58, No. 4, Pp. 1269-1275, 2012.

[5] Zhang Wen and Jiang Meng, "Design of vehicle positioning system based on arm", IEEE, 2011.

[6] N. Ramadan, M.A. Al-Khedher and S.A. Al-Kheder, "Intelligent antitheft and tracking system for automobiles", International Journal of Machine Learning and Computing, Vol. 2, No. 1, 2013.

[7] Yao Jin, "The discussion of Road Traffic Safety Counter measures System Private Science and Technology", 2011.

[8] Z. Yi and Y. Shubo, "MMA series of acceleration sensor principles and application", Medical equipment, 2014.

[9] F. Sposaro and G. Tyson, "I fall: An Android Application for all Monitoring and response", Annual International Conference of the EMBS, Pp. 1367- 1370, 2007.

[10] S.P. Bhumkar, V.V. Deotare and R.V.Babar, "Accident Avoidance and Detection on Highways", International Journal of Engineering Trends and Technology, Vol.3, No. 2, Pp. 247-252, 2012.

[11] Q.V. Vo, G. Lee and D. Choi, "Fall detection based on movement and smart phone technology", IEEE RIVF International Conference on Computing and Communication Technologies, Research, Innovation, and Vision for the Future (RIVF), Pp. 1-4, 2012. 